\title{
El Derecho como argumentación
}

\author{
MANUEL ATIENZA \\ Universidad de Alicante
}

Frente a las concepciones del Derecho como norma, como hecho o como valor (que caracterizan, respectivamente, al normativismo, al realismo jurídico y al iusnaturalismo), se propone aquí un cuarto enfoque que consiste en ver el Derecho como argumentación ( $y$ que cobra especial relevancia en las sociedades democráticas). Sin embargo, no hay una única forma de cntender la argumentación juridica. Aunque conectadas entre sí, en el trabajo se distinguen tres concepciones: la formal, la material y la pragmática o dialéctica; muchas cuestiones que se plantean en el ámbito de la teoría de la argumentación jurídica pueden resolverse -0 aclararse - teniendo en cuenta esa triple perspectiva.

1. El Derecho es, obviamente, un fenómeno muy complejo y que puede contemplarse desde muy diversas perspectivas. En el marco de nuestra cultura jurídica - particularmente la de los paises de Derecho continental-, tres de esos enfoques han tenido, y siguen teniendo, una especial relevancia teórica.

$\mathrm{Al}$ primero de ellos se le puede llamar estructural y da lugar a las diversas formas de normativismo jurídico. Lo que se busca es identificar o encontrar, por decirlo con una metáfora, los componentes del edificio jurídico, con lo que se llega a las normas, a los diversos tipos de normas y, eventualmente, a otros enunciados, como los que contienen definiciones o juicios de valor. Podríamos decir que, al igual que la fotografía de un edificio se plasma en un pedazo de papel, el Derecho así contemplado se reduce a una serie de enunciados, a lenguaje. Si csa fotografía del Derecho es de la suficiente calidad, entonces se podrán observar tanto las partes del edificio - con todo el detalle imaginable - como el edificio en su conjunto, es decir, cómo se ensamblan unos elementos con otros. Para quien asume esa perspectiva, de lo que se trata básicamente es de describir el edificio tal y como es, no de compararlo con un modelo ideal, y menos aún, de construir otro edificio.

El segundo de los enfoques, el realista o sociológico, pone el énfasis en mostrar que el Derecho no es simplemente lenguaje, normatividad, sino también -y sobre todo- comportamiento humano y, en particular, comportamiento judicial. A ese enfoque le interesa también la funcionalidad del edificio, esto es, para qué sirve cada uno de sus elementos y cómo se inserta el mismo en el conjunto del que forma parte (en la sociedad). Pero los realistas - como los normativistas - parten de una distinción tajante entre lo que es y lo que debe ser, y son escépticos con respecto a la posibilidad de hablar con alguna 
objetividad sobre el deber ser. De ahí que se hayan preocupado por explicar o predecir el comportamiento de los jueces (el Derecho en acción), pero no por construir una teoría que permita justificar el desarrollo del Derecho en un cierto sentido. La suya es una visión dinámica e instrumental del Derecho, pero en la que no se dice nada $\longrightarrow$ o nada racionalmente justificado- sobre los fines últimos, sobre los valores jurídicos.

Desde la tercera perspectiva, lo que se destaca es lo que podría llamarse la idealidad del Derecho. Para seguir con la metáfora de la construcción, lo que importa ahora no es ni el edificio ya construido ni el proceso de su construcción, sino los requisitos que tendría que cumplir lo que cabría calificar como edificio modélico (el Derecho justo). Quien elije ese punto de vista (el del Derecho natural en sus muy diversas versiones) se sitúa frente al Derecho, en general, como el crítico que evalúa una determinada obra de acuerdo con los anteriores cánones de idealidad y, en ocasiones, también como el arquitecto que proyecta un edificio, pero se desentiende de las cuestiones de detalle y de los problemas de su ejecución.

Lo que aquí me interesa destacar es la posibilidad de un cuarto enfoque que presupone, utiliza y, en cierto modo, da sentido a las anteriores perspectivas teóricas y que conduce, en definitiva, a ver el Derecho como argumentación (aunque, naturalmente, el Derecho no sea - no pueda ser-sólo argumentación). El punto de partida consiste en considerar al Derecho como una técnica para la solución de determinados problemas prácticos. Se trata, por tanto, de una visión pragmática, dinámica y, en cierto modo, instrumental del Derecho, pero que no contempla el Derecho como un instrumento que pueda ser utilizado para cualquier fin, sino, para decirlo con cierta solemnidad, como un instrumento de la razón práctica. En definitiva, sería la perspectiva de quien no se limita a contemplar el edificio desde fuera, ni a participar en su construcción simplemente como un técnico que opera con una racionalidad de tipo instrumental, sino como alguien comprometido con la tarea de mejorar una obra imperfecta y siempre inacabada, pero no por ello carente de sentido; lo que la dota de sentido es la idea de que el Derecho - el Derecho del Estado democrático- es, al menos incoativamente, un medio poderoso para lograr objetivos sociales valiosos y para hacer que se respeten los principios y valores de una moral racionalmente justificada.

2. Ahora bien, icómo se conecta este cuarto enfoque del Derecho con la argumentación? ¿Por qué lleva en mayor medida que los otros a considerar el Derecho como argumentación? La idea fundamental es que el ideal regulativo del Estado de Derecho es el sometimiento del poder a la razón, no de la razón al poder; ello supone que las decisiones de los órganos públicos deben estar racionalmente fundamentadas, lo que, a su vez, sólo es posible si cabe hablar de criterios que presten algún tipo de objetividad a esa práctica. Dicho en forma breve, el Estado de Detecho exige que el Derecho aparezca esencialmente bajo la forma de razonamiento, de razonamiento práctico justificativo. Vcámoslo. 
El Derecho, en todas sus instancias - legislativa, jurisdiccional, doctrinal, etc.- puede verse como un entramado muy complejo de decisiones vinculadas con la resolución de ciertos problemas prácticos. Pero en el Derecho de las sociedades democráticas, lo que importa no son sólo las decisiones, sino las razones - o cierto tipo de razones - que pueden darse en favor de las decisiones.

Ahora bien, en relación con las decisiones, como en general con las acciones humanas, pueden distinguirse, básicamente, dos tipos de razones: explicativas y justificativas. Las primeras tratan fundamentalmente de dar cuenta de por qué se tomó una determinada decisión - cuál fue la causa que la motivó-, y de para qué —qué finalidad perseguía-. Las scgundas, las razones justificativas, están dirigidas a lograr que la decisión resulte aceptable o correcta. Si se entiende por razonamiento práctico no simplemente un argumento referido a acciones humanas, sino un argumento dirigido a establecer cómo alguien debe comportarse, cntonces razonamiento práctico y razonamicnto justificativo vienen a coincidir. La explicación de la acción humana en términos de causas o de finalidades no da lugar a enunciados de deber ser: la explicación de «ઢ́por qué X realizó $Y$ ?» podrá ser que lo hizo "por causa de $Z$ » o «con el propósito de lograr $F » ;$ pero «X debía - tenía la obligación de- realizar $\mathrm{Y}$ » no es una explicación, sino una justificación de la conducta de $\mathrm{X}$.

La distinción entre explicación y justificación, y entre razonamientos explicativos - teóricos-y justificativos - prácticos-es, obviamente, de gran importancia, pero explicar y justificar son operaciones que muchas veces se entrecruzan: así, del mismo modo que las cuestiones de justificación juegan un papel en la explicación (en muchos casos, lo que explica, por ejemplo, que un juez haya tomado una determinada decisión es -al menos hasta cierto punto- que él la considera justificada), la explicación de las decisiones facilita también la tarea de la justificación (es decir, las posibles razones justificativas aparecen así en forma más explícita).

Cabe decir que el razonamiento jurídico es, en último término, justificativo, pero eso no quita para que las razones explicativas jueguen un papel importante c, incluso, decisivo en muchos casos. Así, motivar una sentencia significa ofrecer una justificación - no una explicación- de la decisión en cuestión, pero eso, en cierto modo, sólo puede hacerse a partir de un esquema - el llamado «silogismo judicial»-, una de cuyas premisas es un enunciado empírico (la afirmación de que ocurrió el hecho $\mathrm{H}$ ), para cuyo establecimiento se necesita contar con razones explicativas adecuadas. Ahora bien, ¿es cierto que la lógica deductiva es la única forma de justificar una decisión? ¿Qué es en realidad lo que justifica la lógica? ¿Y cómo pueden justificarse en general las decisiones?

$\mathrm{Si}$ consideramos que justificar una decisión significa dar razones que la hagan aparecer como correcta o aceptable, entonces cabría decir que este resultado puede lograrse de diversos modos. Básicamente, una decisión puede entenderse justificada de tres maneras: apelando a la autoridad, al procedi- 
miento y/o al contenido. Justificar una decisión en razón exclusivamente de la persona que la dicta - bien se trate de Dios, del monarca, de la asamblea popular, del jurado, etc.- tiene, por así decirlo, el inconveniente de que es escasamente controlable: el único posible objeto de discusión es si la dictó quien podía $\longrightarrow$ debía -dictarla. Si al requisito de la persona se le añade un determinado procedimiento, las posibilidades de discusión aumentan, siempre que se trate de procedimientos controlables racionalmente. Por ejemplo, no hay mucho que discutir frente a un procedimiento como las ordalías o los juicios de Dios o como el que, al parecer, está vigente en la comunidad indígena de Tlacolula, en el Estado de Oaxaca. En esa comunidad existe una institución, la del armado, que encarna el poder político de la misma: el armado -y sus hombres - es quien puede, legítimamente, aplicar la fuerza física en la comunidad. Para resolver el problema de cómo el cargo pasa de un miembro a otro de la comunidad, rige una norma consuetudinaria que establece que el nuevo armado es aquel con el que hayan soñado los dos miembros más viejos de la comunidad. Finalmente, las mayores posibilidades de control $-\mathrm{y}$ de discusión- - existen cuando la justificación de las decisiones se hace depender tanto de la autoridad como del procedimiento y del contenido. $O$, dicho de otra manera, cuando existen normas - como ocurre en nuestros Derechosque regulan quién, cómo y con qué contenido (esto es, dentro de qué limites, con qué objetivos, etc.) puede establecer o aplicar normas jurídicas, y existe también la metanorma que obliga a quienes establecen y aplican normas a dar razones que justifiquen el haber seguido esas normas. Cuanto mayor sea la fuerza de este mecanismo de justificación, tanto mayor también la necesidad de argumentar.

3. ¿Pero qué significa argumentar? ¿Qué es un argumento? El punto de partida para contestar a estas preguntas podría ser éste: en una argumentación - como actividad - y en un argumento - como resultado de esa actividad- existen siempre, cuando menos, estos elementos: 1) un lenguaje, cs decir, argumentar es una actividad lingüística, y un argumento es un producto lingüístico que se plasma en un conjunto de enunciados; 2) una conclusión, esto es, el punto final de la argumentación o el enunciado con que se cierra el argumento; 3 ) una o varias premisas, esto es, el punto de partida de la argumentación o los enunciados con que se abre el argumento, y 4) una relación entre las premisas y la conclusión. Aunque haya diversas formas de entender los argumentos y la argumentación ( $y$, por cierto, cada una de ellas concede más importancia al resultado o a la actividad), parece que esos elementos tienen que darse siempre; eso no quita para que pueda hablarse de un argumento con premisas implícitas (que, claro está, no es lo mismo que premisas inexistentes) o de una argumentación en la que alguno de sus pasos no consiste en una acción lingüistica (pero que siempre podremos plasmar en un lenguaje: como, por ejemplo, la presentación de una prueba en un proceso judicial).

Ahora bien, a pesar de la existencia de estos elementos comunes, no hay una única forma de entender lo que son las argumentaciones y los argumentos. 
Básicamente, creo que puede hablarse de tres concepciones distintas que, a falta de un nombre mejor, llamaré concepción formal, material y pragmática o dialéctica. Cada una de ellas, como ahora veremos, interpreta esos elementos en forma distinta.

3.1. La concepción formal es característica de los lógicos, los cuales definen un argumento, una inferencia, como un encadenamiento de proposiciones: en un argumento deductivamente válido, si las premisas son verdaderas, entonces tambiên lo es necesariamente la conclusión (en virtud de alguna regla de derivación de la lógica). Esta relación de inferencia puede interpretarse en sentido sintáctico, en sentido semántico o, como lo ha hecho últimamente Alchourrón (1995), en sentido abstracto, esto es, construyendo «una concepción general de consecuencia de la cual tanto el enfoque sintáctico como el semántico no sean más que especificaciones diferentes» (p. 36). Pero siempre se tratará de una relación formal, es decir, lo que garantiza el paso de las premisas a la conclusión son reglas de carácter formal, en el sentido de que su aplicación no exige entrar a considerar el contenido de verdad o de corrección de las premisas.

Este carácter formal sigue dándose en las lógicas divergentes (las que se apartan de la lógica estándar), como la llamada lógica de la relevancia. De lo que aquí se trata es de modificar la noción clásica de inferencia deductiva que, al permitir inferencias «irrelevantes» (por ejemplo, de las premisas: «si $\mathrm{X}$ comete tráfico de drogas, debe ser castigado con la pena $\mathrm{P} » \mathrm{y}$ «X ha cometido tráfico de drogas», la lógica estándar permite derivar no sólo « $\mathrm{X}$ debe ser castigado con la pena $P$ », sino también « $X$ debe ser castigado con la pena $P$ o bien debe ser puesto en libertad»), no parece ajustarse bien a las argumentaciones que tienen lugar de hecho, esto es, no se ajusta a nuestras intuiciones. Para evitarlo - para evitar las consecuencias irrelevantes - lo que se hace es restringir la noción de inferencia. Por ejemplo, aceptando sólo una parte de las reglas de la lógica deductiva estándar (las reglas de eliminación). Pero este último es también un criterio formal, para cuya aplicación no se necesita entrar a considerar el contenido de las premisas.

Cabria decir que la lógica no se centra en la actividad de argumentar, en el proceso de la argumentación, sino en los argumentos, en el resultado de la actividad. Lo que la lógica ofrece son esquemas de argumentación, que cabe usar para controlar la corrección de nuestros argumentos. Pero la lógica no describe cómo, de hecho, la gente argumenta. $\mathrm{Y}$ ni siquiera está claro que permita una reconstrucción adecuada de nuestros argumentos. Aunque el leitmotiv de la mayor parte de las lógicas divergentes parece encontrarse en la necesidad de construir lenguajes artificiales, lenguajes formales que reflejen la «logica» interna incorporada a nuestros lenguajes naturales, esto, según lógicos eminentes como Alchourrón, no puede nunca lograrse del todo: aen un sentido importante - ha escrito Alchourrón (1995) - no hay una lógica coherente en el lenguaje natural. El lenguaje corriente no sólo está plagado 
de ambigüedades, vaguedades y toda suerte de imprecisiones significativas que justifican apartarse de él en los procesos de reconstrucción racional, sino que acumula en su seno intuiciones incompatibles que no pueden superarse más que reformándolo, abandonando intuiciones que pueden ser muy sólidas (...) cualquiera que sea la lógica que terminemos privilegiando, ella tendrá que apartarse de las intuiciones básicas incorporadas al esquema de conceptos de los lenguajes corrientes. Esto implica abandonar una idea reconstructiva con pretensiones de resultados unívocos» (pp. 46 y 47 ).

En resumen, desde el punto de vista lógico, más que de argumentos habría que hablar de esquemas de argumentos que se expresan en un lenguaje artificial que no se corresponde exactamente con algún lenguaje natural. Las premisas y la conclusión son enunciados que podrán interpretarse como proposiciones susceptibles de ser calificadas como verdaderas o falsas, pero tambièn como normas que carecen de valores de verdad. $Y$ la relación de inferencia o consecuencia lógica se caracteriza por una serie de propiedades formales, que podrán ser distintas según el tipo de lógica por el que se opte. En el caso de la lógica deductiva estándar, esas propiedades son las de reflexividad generalizada, corte y monotonía. La reflexividad significa que un enunciado se deriva, es consecuencia, de un conjunto de enunciados (premisas) si el primero (la conclusión) está incluido en las premisas: en una deducción, la conclusión no va más allá de las premisas. La propiedad de corte supone que si un enunciado es consecuencia de un conjunto de premisas y de la conjunción de esas premisas más la conclusión se deriva un nuevo enunciado, entonces este nuevo enunciado se deriva también del conjunto incial de premisas: es decir, que las consecuencias de las consecuencias de un conjunto de enunciados son consecuencia del conjunto de partida. Y la propiedad de monotonía, que si un enunciado es consecuencia de un conjunto de premisas, ese enunciado seguirá siendo consecuencia de cualquicr ampliación del conjunto de premisas: o sca, al agregar enunciados a un conjunto de premisas no se pierde ninguna de sus consecuencias.

Naturalmente, este carácter idealizado de la lógica no la priva de virtualidades prácticas, como tienden a creer muchos juristas y algunos teóricos de la argumentación jurídica. La lógica (la lógica deductiva) proporciona un criterio muy importante para controlar la corrección de nuestros argumentos en cualquier empresa racional de que se trate, incluida, por supuesto, la del Derecho. Pero fuera de algunas excepciones - algunas partes de la matemática y la propia lógica - no son criterios suficientes.

3.2. La segunda concepción de la argumentación, la que he llamado concepción material, es la que puede encontrarse, referida al razonamiento jurídico, de alguna forma, en la concepción de la tópica jurídica de Viehweg, en la distinción usual entre justificación interna y justificación externa, o en la teoría de Raz y otros autores de las razones para la acción.

Viehweg (1964) caracteriza la tópica como un ars inveniendi, como una técnica del pensamiento problemático en la que el centro lo ocupa la noción 
de topos o lugar común. Fllo significa que, para él, lo que importa en la argumentación jurídica no es el ars iudicandi, esto es la técnica consistente cn inferir unas proposiciones de otras (como hemos visto, la lógica se ocupa de claborar los criterios que perniten controlar - juzgar- esas inferencias), sino el ars inveniendi, el descubrimiento y examen de las premisas. Para realizar esta última tarea es para lo que se necesita recurrir a los «tópicos», un concepto extraordinariamente equívoco desde sus orígenes en Aristótcles, pero que, en uno de sus sentidos, viene a equivaler a argumentos materiales, esto es, a enunciados de contenido, a premisas materiales que ofrecen un apoyo para la resolución de un problema práctico que no puede ser eludido (lo que Viehweg llama «aporía»).

La distinción entre justificación interna y externa (una distinción que, procedente de Wróblewski - 1971 - es de uso común en la teoría estándar de la argumentación jurídica) apunta también en la misma dirección. La justificación interna se refiere a la validez de una inferencia a partir de premisas dadas; se reduce, pues, a una cuestión de lógica deductiva (la teoría del silogismo judicial). Mientras que la justificación externa se refiere a la justificación de las premisas, lo que no puede hacerse sin recurrir a teorias que no pueden ser ya meramente formales: teorías sobre la interpretación, sobre la valoración de la prueba, etc.

En la concepción del razonamiento práctico de Raz, las premisas no son ya simplemente enunciados, sino razones, y las razones - nos dice Raz de un modo un tanto misterioso - son hechos: «una razón —escribe Raz (1986)es un hecho que por sí mismo basta para imponer cierto curso de acción, siempre que no haya otros factores que la derroten» (p. 28). Un razonamiento - práctico o téórico- no sería puramente una inferencia regida por reglas formales, sino un procedimiento para resolver un conflicto de razones (entre razones prácticas, razones para la acción, o entre razones teóricas, razones para creer en algo). Por eso, Raz considera que la lógica deóntica $\longrightarrow$ o sea, la lógica deductiva aplicada al campo normativo, al campo práctico- no es útil para quien se interesa por el razonamiento práctico, «porque olvida por completo los problemas presentados por los conflictos de razones (...). Pero la principal tarea de la teoría de la razón práctica - añade Raz- es establecer lo que tenemos (prima facie) razones para hacer y cómo resolver los conflictos de razones y establecer aquello que debemos hacer, tomando todo en consideración» (p. 28). La teoría de Raz del razonamiento práctico se resuelve por eso en una teoría de las razones para la acción, es decir, en una teoría sobre los diversos tipos de razones para la acción existentes y los modos de resolver los conflictos entre esas razones. La teoría alcanza en Raz niveles de gran abstracción - probablemente no exenta de alguna oscuridad-, pero no es una teoría formal, o al menos no lo es en el sentido de la lógica, en cuanto que desemboca en -o presupone- una determinada filosofía moral y política. 
Esta manera de entender el razonamiento práctico es también la que puede encontrarse en autores como Dworkin, Nino o Summers. Este último, por ejemplo, ha elaborado una tipología de lo que él considera que son las «buenas razones" que pueden encontrarse en los casos resueltos por los jueces del common law: razones sustantivas, autoritativas, factuales, interpretativas y críticas. Las razones sustantivas, a su vez, pueden ser razones finales (por ejemplo, la «promoción de la armonía familiar») o razones de corrección (por ejemplo, la «obligación de restituir por enriquecimiento injusto»). En el trabajo al que me estoy refiriendo, Summers (1978) pretende efectuar una reconstrucción racional (no una descripción psicológica) del proceso mediante el cual los jueces identifican, construyen y evalúan las razones sustantivas (p. 59).

3.3. Finalmente, la tercera concepción, la concepción pragmática o dialéctica, considera la argumentación como un tipo de acción -o de interacciónlingüistica. La argumentación es un acto de lenguaje complejo que tiene lugar en situaciones determinadas; en general, podría decirse que en el contexto de un diálogo (con otro o con uno mismo), cuando aparece una duda o se pone en cuestión un enunciado (de caracter teórico, práctico, etc.) y se acepta que el problema ha de resolverse por medios lingüisticos (por tanto, sin recurrir a la fuerza física o a otros tipos de presiones externas al discurso). La argumentación es, pues, vista aquí básicamente como una actividad, como un proceso, cuyo desarrollo está regido por determinadas reglas de comportamiento (de comportamiento lingüístico) de los sujetos que intervienen en la misma. Ésta es la conccpción que cabe encontrar en muchas teorías contemporáneas de la argumentación, como la de Toulmin (1958) o Habermas (1987), y es, básicamente, el modelo al que obedecc la nucva retórica de Perelman (Perelman y Olbrecht-Tyteca, 1989) o la teoría de la argumentación jurídica de Alexy (1989).

En síntesis, podría decirse que la argumentación, de acuerdo con esta tercera perspectiva, consiste también en lenguaje, pero lo que aparece destacado es el aspecto pragmático del lenguaje, y de ahí que la argumentación sea inconcebible haciendo abstracción de los sujetos que argumentan. La argumentación avanza, es posible, en la medida en que los participantes se van haciendo concesiones; inferir consiste aquí en el paso de unos enunciados a otros mediante la aceptación, el consenso; para cada interviniente en el proceso, funcionan como premisas los enunciados cuya aceptación pueda darse por supuesta o por alcanzada en cada momento del proceso; y la conclusión es lo que se pretende sea aceptado por el otro.

4. El hecho de que se trate de tres concepciones distintas de la argumentación no significa exactamente que sean incompatibles entre sí. Más bien me parece que habría que tratarlas como complementarias, en el sentido, por ejemplo, de que una teoría adecuada de la argumentación jurídica no puede dejar de considerar ninguno de esos tres enfoques. La razón es que el Derecho en cuanto práctica racional, y en particular el Estado de Derecho, el Estado 
constitucional, presupone - - o implica - no sólo valores de tipo formal (ligados con la idea de previsibilidad), sino también de tipo material (vinculados a las nociones de justicia o de verdad) y de tipo pragmático o político (conectados a la noción de aceptación). Por lo demás, me parece que hay no pocas cuestiones que se plantean en el ámbito de la argumentación jurídica y de la teoría del Derecho, que podrían clarificarse teniendo en cuenta esas tres perspectivas: por ejemplo, la distinción entre el contexto de descubrimiento y el contexto de justificación; el criterio de demarcación entre las buenas y las malas argumentaciones; la clasificación de las falacias jurídicas, de los malos argumentos que parecen buenos y que con cierta frecuencia se emplean en las diversas instancias de elaboración, sistematización y desarrollo del Derecho; o la conexión entre las teorías de la argumentación y de la interpretación jurídica (Atienza, 1997 y 1998). Aquí me referiré únicamente a la primera de esas cuestiones.

La distinción entre el contexto de descubrimiento y el contexto de justificación procede, como se sabe, del ámbito de la teoría de la ciencia (de la epistemología neopositivista), pero se ha trasladado al campo de la argumentación jurídica y, en particular, al de la decisión judicial. Así, una de las características de lo que en ocasiones he llamado «teoría estándar de la argumentación jurídica» (Atienza, 1989) cs que reduce el estudio de la argumentación jurídica al contexto de la justificación.

En el ámbito de la teoría de la ciencia, la distinción (que no es unánimemente aceptada ni quizás tampoco entendida de la misma manera) estaría entre lo que son criterios de validación de una teoría científica (el contexto de la justificación) y el proceso mediante el que se llega a formular, a descubrir, una determinada teoría (el contexto del descubrimiento). De lo que se trata es de trazar un criterio de delimitación entre el análisis lógico de una teoría científica y las investigaciones empíricas de carácter psicológico, sociológico o histórico. Un ejemplo paradigmático de la distinción puede serlo el descubrimiento de la estructura del benceno por el químico Kekulé. Al parecer, a Kekulé se le ocurrió la idea de que las moléculas del benceno estaban dispuestas en forma de un anillo exagonal contemplando las llamas en la chimenea de su casa. Ahora bien, la forma en que se produjo ese descubrimiento no tiene nada que ver - se afirma-con la cuestión - que pertenecería al contexto de la justificación - de si la teoria, o la hipótesis, en cuestión resulta o no científicamente aceptable. Las reglas del método científico sólo rigen en este último campo, pero no en el primero: no existe - de acuerdo con los filósofos neopositivistas- una «lógica del descubrimiento».

Trasladada al campo de la argumentación jurídica, la distinción se ha utilizado para oponerse a ciertos teóricos del Derecho - algunos realistas radicales, como Frank- que consideran que las decisiones jurídicas - las decisiones judiciales- no pueden ser justificadas, ya que los jueces --o los juradostoman esas decisiones en forma irracional -0 arracional-; la motivación de 
las sentencias no sería más que una "racionalización» de una operación que no obedece en absoluto al esquema de la lógica, al silogismo judicial. Quienes sostienen esto último, se dice, estarían confundiendo el contexto de descubrimiento y el contexto de justificación. «Es posible que, de hecho, las decisiones se tomen, al menos en parte, como ellos sugieren, es decir, que el proceso mental del juez vaya de la conclusión a las premisas e incluso que la decisión sea, sobre todo, fruto de prejuicios; pero ello no anula la necesidad de justificar la decisión, ni convierte tampoco esta tarea en algo imposible; en otro caso, habria que negar también que se pueda dar el paso de las intuiciones a las teorías científicas o que, por ejemplo, científicos que ocultan ciertos datos que se compadecen mal con sus teorías estén por ello mismo privándolas de justificación» (Atienza, 1991, 26).

Como ha señalado convincentemente Tecla Mazaresse (1996), trasladar la distinción contexto del descubrimiento/contexto de la justificación al campo de la argumentación jurídica, de la decisión judicial, resulta problemático por diversas razones. Una es que la propia distinción, a propósito de las teorías científicas, no deja de ser cuestionable: puede entenderse de diversas maneras y cabe incluso negar que la distinción en cuanto tal tenga sentido. Otra es que entre una teoría científica y una decisión práctica existen diferencias notables, de manera que lo que vale para un caso podría no valer para el otro; piénsese, por ejemplo, en que cuando se habla de ejustificación» a propósito de una teoría científica nos estamos refiriendo a algo muy distinto a una «justificación práctica»: así, en relación con la conducta humana, las teorías científicas ofrecen esquemas explicativos, no justificativos de la conducta. Sin embargo, el caracter problemático de la distinción no significa, en mi opinión, que la misma sea inútil, o que deba abandonarse. Se trata, a mi juicio, de una distinción importante, pero cuya adecuada utilización exige ser consciente del triple enfoque de la argumentación a que antes me refería.

Si la argumentación se ve desde el punto de vista lógico, la distinción en cuestión parece ciertamente pertinente: la lógica, como bemos visto, se centra en la argumentación como resultado, en el paso, por ejemplo, de la premisa fáctica ( $« X$ ha realizado la acción $F »)$ y normativa ( Quien realiza $F$ debe ser condenado con la pena $P$ ») a una conclusión («Debo condenar a $X$ a la pena $P »)$ que, por lo demás, no es la decisión en cuanto tal ( $«$ Condeno a $X$ a la pena $P »$ ); lo que importa aquí no es cómo se pasa de las premisas a la conclusión, sino establecer si un determinado paso es o no lógicamente correcto. Y así, por ejemplo, el hecho de que, desde el punto de vista psicológico, se alcance primero la conclusión y luego se formulen las premisas mediante un mecanismo de «racionalización» a posteriori, no afecta para nada a la cuestión de si el paso - la inferencia- está o no justificada lógicamente; la justificación lógica es de carácter puramente formal:

Si las cosas se ven en términos del "razonamiento práctico», una de las versiones de lo que antes habíamos llamado concepción material de la argu- 
mentación, la distinción pierde quizäs bastante de su nitidez, puesto que aquí sí que interesa ya el proceso de la argumentación. Por supuesto, cabe hacer una distinción entre el proceso psicológico del balance de razones y lo que sería una «reconstrucción racional» del mismo, pero a la hora de evaluar el resultado del balance - el juicio práctico en que el mismo se resuelve - parecería que el hecho de que el razonador haya tenido la actitud psicológica adecuada resulta relevante: el contexto de la justificación no es, pues, aquí independiente del de descubrimiento.

Finalmente, la distinción prácticamente desaparece si la argumentación se contempla desde la tercera perspectiva: la concepción pragmática o dialéctica. El criterio de evaluación de los argumentos es aquí inseparable del propio comportamiento de los participantes y/o de los destinatarios. La distinción que cabe trazar aquí entre lo empírico y lo normativo tiene lugar en el plano del comportamiento, entre el comportamiento real y el ideal de los sujetos; de esta forma, se podrá distinguir entre un argumento persuasivo y un argumento convincente $o$, dicho de otra manera, entre un argumento que es eficaz, que es aceptado, de hecho, y un argumento que debería ser eficaz, que debería ser aceptado (o, si se quiere, que es aceptado por una comunidad ideal de seres).

\section{BIBLIOGRAFÍA}

Alchourrón, Carlos E.: «Concepciones de la lógica», en Enciclopedia Iberoamericana de Filosofia, vol. 7, Lógica, CSIC.Trotta, Madrid, 1995.

AlExy, Robert: Teoria de la argumentación juridica, Centro de Estudios Constitucionales, Madrid, 1989.

Atienza, Manuel: Las razones del Derecho. Teorias de la argumentación jurídica, Centro de Estudios Constitucionles, Madrid, 1991.

- «Estado de Derecho, interpretación y agumentación", en Anuario de Filosofia del Derecho, XIV, 1997.

- «A propósito de la argumentación jurídica», en Doxa, núm. 21, vol. II, 1998.

HaBERMAS, Jürgen: Teoría de la acción comunicativa, Taurus, Madrid, 1987.

MAZzAREsse, Tecla: Fome di razionalità delle decisioni giudiziali, Giapichelli, Turín, 1996.

Perelman, Chaïm, y Olbrecht-Ty'tca, Lucie: La nueva retórica. Tratado de la argumentación, Gredos, Madrid, 1989.

RAz, Joseph: «Introducción» al vol. colectivo Razonamiento práctico (compilación de J. Raz), Fondo de Cultura Económica, México, 1986.

Summers, Robert S.: «Two Types of Substantive Reasons: The core of a theory of common-law justification», en Comell Law Review, núm. 63, 1978.

Toulmin, Stephen E.: The Uses of Argument, Cambridge University Press, 1958.

VIEHwEG, Theodor: Tópica y jurispridencia, Taurus, Madrid, 1964.

WRÓblewskı, Jerzy: «Legal Decision and its Justification», en Le raisonnement juridique (ed. de H. Hubien), Bruselas, 1971. 